\title{
How to interpret a paediatric blood culture
}

\author{
Shaun O'Hagan, ${ }^{1}$ Peter Nelson, ${ }^{2}$ Lynne Speirs, ${ }^{1}$ Paul Moriarty, ${ }^{1}$ \\ Peter Mallett (D) ${ }^{1}$
}

- Additional material is published online only. To view, please visit the journal online (http://dx.doi.org/10.1136/ edpract-2020-321121).

'Department of Paediatrics, Royal Belfast Hospital for Sick Children, Belfast, UK

${ }^{2}$ Clinical Microbiology, Belfast Health and Social Care Trust, Belfast, UK

\section{Correspondence to}

Dr Shaun O'Hagan, Royal Belfast Hospital for Sick Children, Belfast BT12 6BA, UK; shaun.o'hagan@nhs.net

Accepted 8 February 2021 Published Online First 26 February 2021

\section{Check for updates}

(c) Author(s) (or their employer(s)) 2021. No commercial re-use. See rights and permissions. Published by BMJ.

To cite: O'Hagan S, Nelson P, Speirs L, et al. Arch Dis Child Educ Pract Ed 2021;106:244-250.

\begin{abstract}
Blood culture is one of the most important diagnostic tests in medicine, considering the significant morbidity and mortality associated with bloodstream infection (BSI). However, it is an often misused and misinterpreted test in everyday paediatric practice. In this article, we explore the evidence related to paediatric blood cultures, with the aim of providing clear and clinically-relevant recommendations for its judicious use.
\end{abstract}

\section{INTRODUCTION}

Blood culture (BC) is routinely performed in the assessment of children with fever; however, there remains considerable ambiguity as to best practice for optimal timing, adequate blood volume and correct interpretation of results. ${ }^{1}$

With increasing global antimicrobial resistance, it has never been more important to better understand the critical role of $\mathrm{BC}$ in antimicrobial stewardship by minimising inappropriate antibiotics, reducing length of hospital stay, improving patient safety and preserving existing antibiotics for future generations. ${ }^{2}$

In this article, we will follow the $\mathrm{BC}$ journey from the patient's bedside to the microbiology laboratory and back. Our aim is to provide a pragmatic guide to aid clinician understanding, focus rationale for performing and assist in the interpretation of results.

Laboratory functions are grouped into three phases: ${ }^{3}$

1. Preanalytical phase: time from when a test is performed to when it arrives in the laboratory.

2. Analytical phase: handling and analysis of the sample.

3. Postanalytical: what happens after a result is obtained, including reporting.

\section{PREANALYTICAL PHASE}

Case 1: A previously well 3-year-old boy attends the emergency department with a 2-day history of fever $\left(38^{\circ} \mathrm{C}\right)$ and an erythematous tender rash on his right thigh. The working diagnosis is moderate cellulitis and you plan to prescribe oral antibiotics. You wonder, should I send a BC?

Diagnostic stewardship: testing the right patients for the right reasons

Before obtaining a $\mathrm{BC}$, it is crucial to establish the pretest probability of bloodstream infection (BSI). ${ }^{3}$ Selecting patients with reasonable suspicion of BSI improves diagnostic yield ${ }^{4}$ and helps when interpreting the significance of a positive culture. If you do not suspect BSI, do not send a BC. ${ }^{5}$

This is especially important, given that multiple studies have found the majority of positive paediatric BCs are contaminants. ${ }^{6}$ When obtaining a $\mathrm{BC}$, careful skin preparation and aseptic technique is vital to reduce the risk of contamination with skin commensals. ${ }^{7}$

When to perform a BC can be perceived as an unclear issue for clinicians. In essence, it is important to consider

- The clinical presentation and severity of condition.

- Probable source of infection.

- Medical history.

- Relevant risk factors for the development of BSI.

If there is a clinical impression of mild infection and low probability of BSI, performing a $\mathrm{BC}$ is rarely indicated. The British Society for Antimicrobial Chemotherapy has recently published detailed clinical pathways for common paediatric infections. These provide evidence-based, focused guidance on presenting features, diagnostics, interpretation of investigations and management advice. Clinicians caring for children in any healthcare setting should familiarise themselves with these pathways, which promote sage diagnostic and antimicrobial stewardship principles. ${ }^{8}$ 
Invasive bacterial infection (IBI) is very rare in previously well children, and two-thirds of communityacquired infections occur in children with pre-existing comorbidity. ${ }^{9}$ BSI is often secondary to focal infection at another site, rather than a primary intravascular infection. However, this can occur and is more common in neonates and neutropenic patients. ${ }^{2}$

BC is extremely low-yield in uncomplicated community-acquired pneumonia and soft tissue infections. ${ }^{10}$ Furthermore, isolated pathogens in these infections are often susceptible to first-line antibiotics, meaning empiric antibiotic treatment is generally most appropriate. No single temperature threshold is adequately sensitive to exclude IBI and forego diagnostic evaluation. When determining pretest probability of BSI, height of fever should be considered in the wider clinical context, rather than in isolation. ${ }^{11}$ Later in the article, we review risk factors and clinical predictors of BSI; these can be used as a guide to determine which children should have BCs sent.

\section{Anaerobic BCs}

Anaerobic bacteria are typically fastidious, slowgrowing and difficult to culture. Anaerobic BSI is significantly less common in previously well children, compared with the adult population. ${ }^{2}$ The practice of obtaining routine anaerobic BC remains contentious, and there is broad variation in practices among paediatric centres globally. Current Infectious Diseases Society of America guidelines recommend obtaining both aerobic and anaerobic BCsfor adult and paediatric patients with suspected BSI or septic shock. ${ }^{12}$

Many of the common commercial BC detection systems (eg, BacT/Alert (BioMérieux) or BACTEC (Becton Dickson)) can provide suitable environmental and nutritional conditions for the growth and detection of some of the more common, clinically relevant anaerobic micro-organisms, in addition to aerobic and some fungal species. For this reason, many paediatric centres advise a more pragmatic approach to a small-volume paediatric blood sample, which involves preferentially inoculating one aerobic BC bottle. Nevertheless, many of these centres do promote the judicious use of separate anaerobic BC in paediatric patients at higher risk of invasive anaerobic infection. These include immunocompromised children, those with head and neck, intra-abdominal or pelvic infections, or severe sepsis. ${ }^{212}$

Case 2: A 5-day-old preterm baby has new temperature instability and feed intolerance. You are concerned about necrotising enterocolitis. You obtain $1.5 \mathrm{~mL}$ of blood and wonder: what volume is sufficient for $B C$ ?

$\mathrm{BC}$ volume: you just can't get enough!

Due to the inherent difficulty of paediatric venepuncture, BCs are frequently sent alongside cannulation 'just in case'. Likewise, in a febrile child that may need BC sent (based on clinical progress or inflammatory markers), they are often sent pragmatically to avoid repeated venepuncture. Unfortunately, this means cultures are often inoculated with small volumes, and a negative culture is practically inevitable in a significant proportion. $^{13}$

Numerous factors contribute to BC sensitivity, including

- Blood volume.

- BSI periodicity (transient, intermittent or continuous).

- Causative organism.

- Bacteria density in the bloodstream. ${ }^{14} 15$

However, it is widely accepted that blood volume is the single most important factor ${ }^{1}$ and the main variable determining $\mathrm{BC}$ sensitivity and specificity. ${ }^{16}$ In the adult population, each additional millilitre of blood cultured has been associated with an almost 3\% increase in microbial yield. ${ }^{17}$ Increased BC yield with larger volumes has also been observed in children, with one study reporting an increase from approximately $2 \%$ among cultures inoculated with $\leq 1 \mathrm{~mL}$, up to $>6 \%$ among cultures with $>4 \mathrm{~mL}^{18}$

Obtaining a larger volume not only increases probability of detecting a pathogen but also inversely correlates with bacterial time to detection. ${ }^{14}$ Volumes of $<0.5 \mathrm{~mL}$ especially reduce culture sensitivity for fastidious organisms, such as Haemophilus influenzae, Streptococcus pneumoniae and Neisseria meningitidis. ${ }^{1}$ Additionally, risk of contamination and false-negative cultures are elevated by inadequate blood volume. ${ }^{19}$

BC volume should be proportional to the child's total blood volume and weight. While there remains no absolute consensus, and established practice often involves obtaining much lower volumes than $\mathrm{BC}$ manufacturer recommendations, the minimum $\mathrm{BC}$ volume we should aim to collect is the child's age in millilitre, with special consideration for very small babies, where no more than $4 \%$ of total blood volume should be collected. ${ }^{4}$ Our suggested minimum volumes are as follows.

Case 3: A 6-year-old girl is being treated for pyelonephritis. She has been on intravenous antibiotics for 2 days but remains febrile $\left(>39^{\circ} \mathrm{C}\right)$. Her BC on admission (prior to antibiotics) is sterile to date. She is due for further blood tests, and you wonder: should I send a repeat $\mathrm{BC}$ ?

The best time to culture for BSI is 'as early as possible' in the febrile illness, ${ }^{13}$ with sample collection guided by patient acuity and necessity to commence antibiotics. $^{20}$

In children already receiving antibiotics, there is considerably reduced detection of bacteria by culture

Suggested minimum BC volumes dependent on patient age and weight

$<1 \mathrm{~kg}: ~ 0.5 \mathrm{~mL},<5 \mathrm{~kg}: 0.5-1 \mathrm{~mL},<1$ year: $1 \mathrm{~mL}, 2-5$ years: -

$2-5 \mathrm{~mL}, 5-16$ years: $5-15 \mathrm{~mL}$ 
and, to a lesser extent, by nucleic acid detection methods. ${ }^{21}$ Several studies show that antibiotics prior to $\mathrm{BC}$ sampling decreases the rate of culture positivity by $45 \%-50 \% .{ }^{172}$ In any child already receiving antibiotics, BC should be obtained immediately prior to an antibiotic dose, when antibiotic concentration will be at its lowest. ${ }^{2}$

A common paediatric practice is to obtain $\mathrm{BC}$ during or immediately following fever, in the hopes of increasing diagnostic yield. However, research has demonstrated that fever has no influence on the likelihood of obtaining a positive result. ${ }^{22}$

\section{ANALYTICAL PHASE}

In the UK and Ireland, it is routine to use a fully automated and continuously monitored commercial system to process BC. A blood:broth ratio of $1: 15$ is essential to inhibit the antibacterial effects of normal blood. ${ }^{23}$ Given the acknowledged challenges of paediatric venepuncture, this requirement suggests that many paediatric samples may have a suboptimal ratio and may contribute to a false negative. Additionally, exponential growth within the $\mathrm{BC}$ media means that contamination with just one bacterial colony can result in a false positive.

Samples should be loaded within a maximum of 4 hours to ensure optimal growth. The importance of this practice is highlighted by a neonatal study, which found that mean total time to positivity (TTP) (time from BC inoculation to positive culture), for all isolated organisms at all concentrations, was significantly increased with increasing delay in $\mathrm{BC}$ entry into an automated system. ${ }^{24} \mathrm{~A}$ temperature of $35^{\circ} \mathrm{C}-$ $37^{\circ} \mathrm{C}$ for $5-7$ days is recommended. If a slow-growing organism is suspected, the incubation time should be increased, and it is vital that clinical teams provide this information.

The automated system continuously agitates, incubates and monitors the $\mathrm{BC}$ bottle. A sensor at the base of the bottle detects hydrogen ions generated from the reaction of water and carbon dioxide, which is produced by growing organisms. This results in a colour change in the indicator contained in the sensor. An optical sensor monitors this change and will flag the bottle as positive once one or more criteria are met:

1. Acceleration of $\mathrm{CO}_{2}$ production.

2. The rate of $\mathrm{CO}_{2}$ production exceeds a predetermined threshold.

3. Initial $\mathrm{CO}_{2}$ concentration is above a preset value.

A positive bottle will then be removed from the system and gram stain, along with subculturing, performed.

\section{Gram staining}

This test allows classification of bacteria based on the constituents of the cell wall. The morphology of the bacteria is also used to help with further identification. A sample of blood from a positive BC is mixed with saline and streaked on a microscope slide. It is then dried on a heat plate and stained using the Gram stain method. The slide is examined by microscopy and categorised by the organism's ability to retain the stain.

Gram-positive organisms will appear violet as they have a thick layer of peptidoglycan in the cell wall allowing retention of the crystal violet stain.

Gram negative organisms possess a thin layer of peptidoglycan between two membranes and cannot retain crystal violet. As a result, they are stained pink by a fuchsin counterstain.

\section{POSTANALYTICAL PHASE}

Case 4a: A 1-year-old boy is admitted with fever $\left(38^{\circ} \mathrm{C}\right)$ and poor oral intake. The presumptive diagnosis is a viral upper respiratory tract infection. On day 2 of admission, he is well and afebrile, but remains on intravenous antibiotics. At handover, you ask whether a negative BC excludes BSI and, if not, why it is common practice to wait $\mathbf{4 8}$ hours before stopping intravenous antibiotics? A negative BC does not always exclude BSI, especially if a small inoculum was obtained. In any child with high pretest probability and negative $\mathrm{BC}$ to date, and particularly in those with suspected sepsis, it is worth considering repeat culture and targeted PCR testing; such as for meningococcus, pneumococcus and group B Streptococcus in neonates.

Waiting until 48 hours to stop antibiotic therapy in all children is an outdated approach. Research shows that $90 \%$ of bacteria will have grown by 24 hours and $95 \%$ by 36 hours. ${ }^{25}$ In children with low BSI suspicion, stopping antibiotics at 24-36hours with good safety-netting advice avoids unnecessary hospitalisation without jeopardising patient safety. ${ }^{4}$

Case 4b: Later that day, the microbiology registrar informs you that this patient has grown Gram-positive cocci in clusters/clumps, from an aerobic peripheral BC at 32 hours. You review the child, who remains well and afebrile, and ask the consultant, what is the significance of this positive $\mathrm{BC}$ ?

Species Identification \& Clinical Implication

Few micro-organisms are unequivocal pathogens, and the significance of any positive culture should be interpreted in the context of the clinical scenario. Several questions should be considered:

1. What is the TTP?

2. Is it a pure culture or mixed growth?

3. Is the micro-organism isolated on more than one occasion (persistent positive culture)?

4. Is the organism a common commensal (could it represent contamination)?

5. Does the presence of this organism fit with the clinical picture? $?^{2}$

It is important to note that TTP is inversely proportional to the bacterial density in blood, with infected blood typically having a higher concentration of bacteria than contaminated blood. ${ }^{26}$ As such, any 


\begin{tabular}{ll}
\hline Table 1 & Bloodstream infection risk factors ${ }^{35}$ \\
\hline Prematurity & Young age \\
\hline Immunosuppression & $\begin{array}{l}\text { Indwelling catheter (eg, Hickmann } \\
\text { line and ventriculoperitoneal shunt) }\end{array}$ \\
Neutropenia & $\begin{array}{l}\text { Serious illness requiring intubation } \\
\text { and ventilation }\end{array}$ \\
Eczema (and other inflammatory skin conditions)
\end{tabular}

culture yielding growth within a short duration has a higher probability of being a true pathogen. ${ }^{27}$

Factors indicative of true BSI include suggestive history and clinical examination, shorter TTP, risk factors for BSI (See table 1), fever and raised inflammatory markers. ${ }^{28}$

A suggested approach to positive BC interpretation is demonstrated in figure 1 , with common paediatric species summarised.

\section{Contaminants}

Contaminants create diagnostic conundrum and lead to unnecessary antibiotics. They expose children to antibiotic side effects, contribute to the selection of antibiotic-resistant bacteria and cause distress through repeated cannulation and culture sampling. ${ }^{26}$ Contaminants also have financial implications, increasing length of hospital stay, laboratory costs and antibiotic duration. ${ }^{29}$ With any positive $\mathrm{BC}$, if the diagnosis of true BSI is in doubt, an accepted strategy is to obtain repeat samples. ${ }^{18}$ If multiple BCs are taken and only one is positive, or different bacteria are isolated from repeat culture, this likely represents contamination.

\section{Persistent positive BC}

When the same micro-organism is repeatedly isolated from repeat cultures, thorough daily clinical re-evaluation is warranted. There must be active attempts for source identification and concerted efforts to achieve source control. A suggested approach is provided in figure 2 .

\section{Pathogens}

Modern automated BC systems are highly effective at identifying the most prevalent bacterial bloodstream pathogens. The most common pathogens, categorised according to age, are summarised in table 2. Importantly, Staphylococcus aureus, Candida spp. and Gramnegative bacilli isolated from $\mathrm{BC}$ are predominantly significant organisms, and very careful consideration should be given before discounting these as contaminants. $^{2728}$

\section{Case 5: A 9-year-old boy, with background of previous cardiac surgery, presents with a 6-week history of malaise, night sweats and intermittent fever $\left(38^{\circ} \mathrm{C}\right)$. The clinical suspicion is subacute bacterial endocarditis (SBE). The infectious diseases team recommend taking three sets of appropriate- volume $(\sim 10 \mathrm{~mL})$ peripheral BCs over the next 24 hours. You consider which organisms are particularly difficult to detect on BC?}

\section{Pathogen identification}

Where there is a clinical suspicion of SBE, international guidelines recommend at least three $\mathrm{BC}$ sets should be collected, each containing $10 \mathrm{~mL}$ of blood,

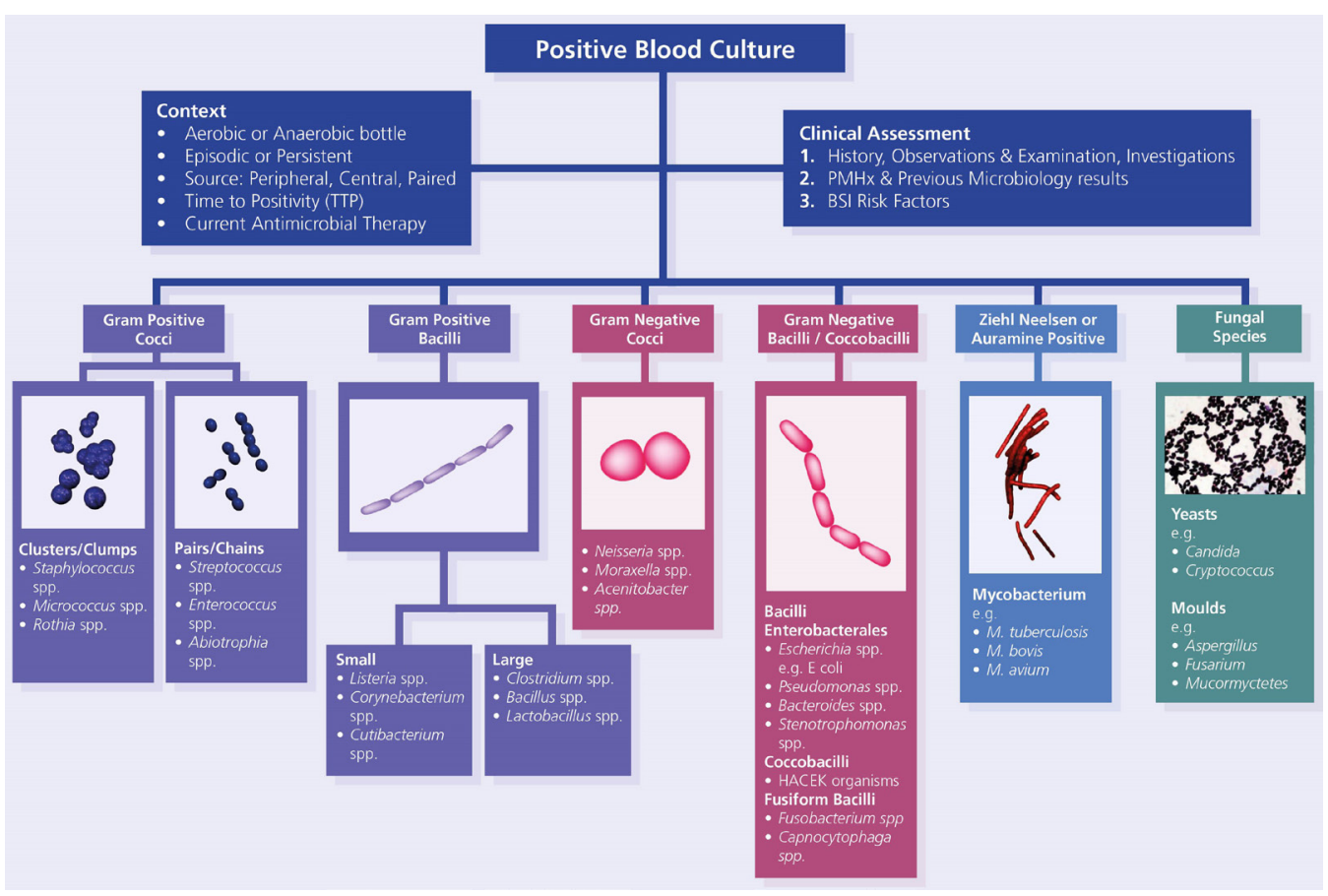

Figure 1 Holistic approach to positive blood culture interpretation. BSI, bloodstream infection; HACEK, Haemophilus, Aggregatibacter, Cardiobacterium, Eikenella, and Kingella species, $\mathrm{PMHx}$, past medical history. 


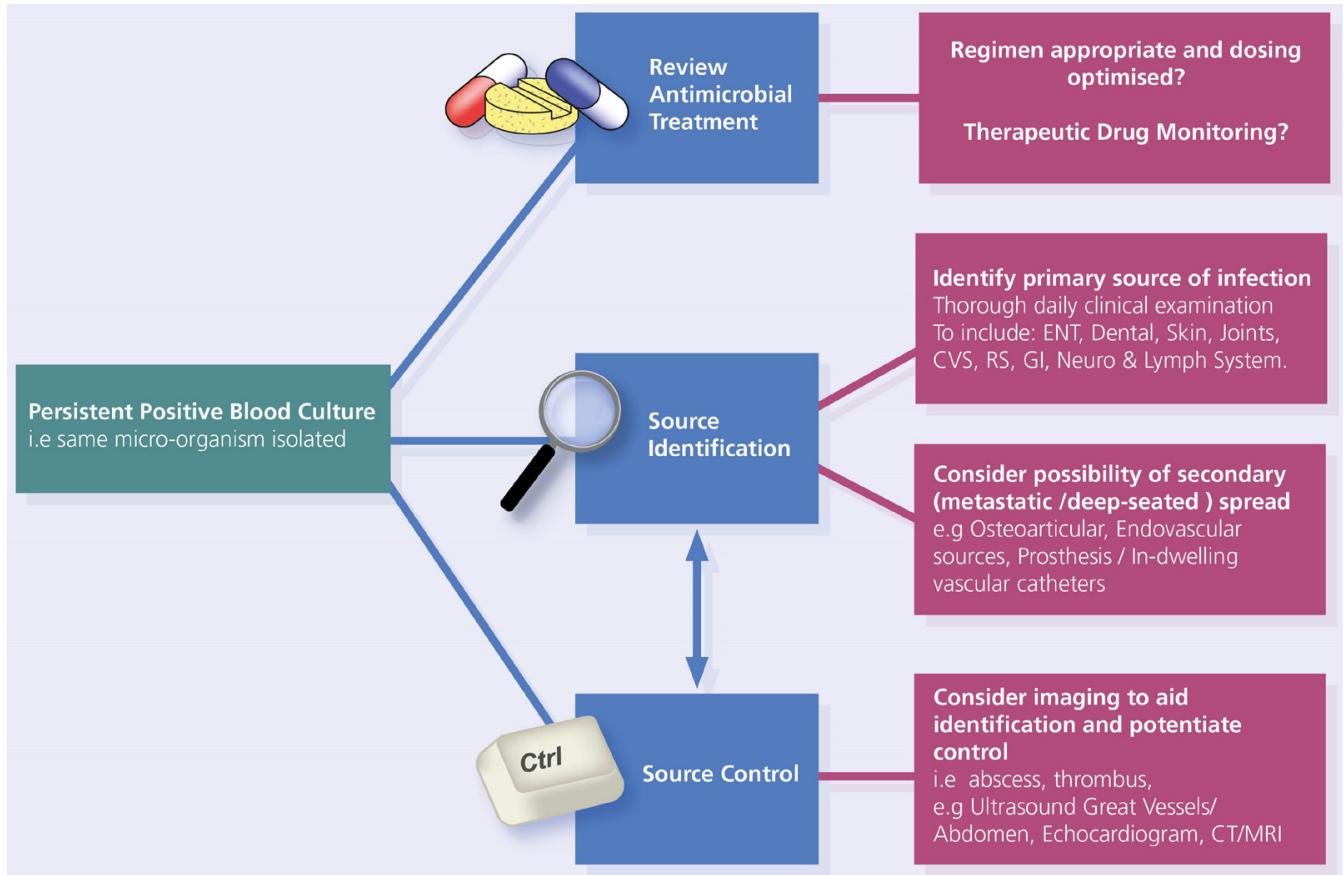

Figure 2 Suggested approach to persistent bacteraemia. ENT, ears, nose and throat; CVS, cardiovascular system; RS, respiratory system; Gl; gastrointestinal.

with samples incubated in both aerobic and anaerobic culture bottles. Particularly in stable patients with clinical suspicion of SBE, antibiotics should be withheld and cultures obtained prior to antibiotic administration to maximise likelihood of identifying a causative organism. Once an organism is identified, BC should be repeated $48-72$ hours after starting antibiotics to assess the effectiveness of treatment. ${ }^{30}$

Traditionally, growth characteristics and a range of biochemical tests were used to identify the species of bacteria cultured. Matrix assisted laser desorption/ ionisation time of flight is a semiautomated form of mass spectrometry which allows rapid identification of cultured organisms, following detection of growth in BC. This has revolutionised laboratory identification methods. ${ }^{2}$ It has also been shown to be cost effective and to shorten the time until appropriate antibiotic therapy in paediatric inpatients. ${ }^{31}$ This assay can, however, misidentify uncommon and fastidious

\begin{tabular}{ll}
\hline $\begin{array}{l}\text { Table } 2 \\
\text { children }{ }^{1}\end{array}$ & Common bloodstream infection pathogens in \\
\hline Age group & Most common organisms \\
\hline Infants $<3$ months & $\begin{array}{l}\text { Escherichia coli, Streptococcus } \\
\text { agalactiae and Staphylococcus aureus }\end{array}$ \\
Children $>3$ months & $\begin{array}{l}\text { S. aureus, S. pneumoniae, Neisseria } \\
\text { meningitidis }\end{array}$ \\
Adolescents & $\begin{array}{l}\text { N. meningitidis } \\
\text { Immunocompromised children }\end{array}$ \\
& $\begin{array}{l}\text { All aforementioned, including } \\
\text { Pseudomonas aeruginosa and Candida } \\
\text { spp. }\end{array}$ \\
\hline
\end{tabular}

organisms, and results should be analysed in conjunction with the clinical presentation. ${ }^{32}$

Bacterial identification directly from positive BC bottles by PCR is also becoming increasingly available, with systems such as the FilmArray Blood Culture Identification Panel (Biomerieux). Early microorganism identification can highlight likely contaminants and allows rapid antibiotic rationalisation. This helps to reduce the use of broad-spectrum antibiotics, improves antibiotic stewardship and can facilitate earlier discharge from hospital. ${ }^{33}$

Fastidious organisms require different growth media, temperatures and atmospheric conditions compared with those routinely used to culture organisms. For example, Haemophilus spp. (which can cause SBE) require two additional growth factors to aid culture. This once again reiterates the importance of providing comprehensive clinical information, including the suspicion of SBE, when requesting BCs.

Typically, it takes 24-48 hours from detecting a positive $\mathrm{BC}$ to ascertaining antibiotic susceptibility. Antimicrobial sensitivity testing is performed using standardised antibiotic impregnated discs and automated broth microdilution systems, which analyse the minimum concentration of an antibiotic required to inhibit growth.

\section{Novel identification methods: bacterial 16S PCR}

If $\mathrm{BC}$ has not yielded a pathogen and BSI is strongly suspected, 16S PCR testing can be performed. All bacterial ribosomes have a highly conserved $16 \mathrm{~S}$ subunit with variable regions specific to different 
species. Using 16S PCR and DNA sequencing, any bacterial DNA in the sample can be amplified and help identify the genus and species. This is particularly useful in culture-negative endocarditis and can be applied to any sterile sample that has not been cultured an organism. ${ }^{34}$ However, availability of this method is predominantly limited to reference laboratories. Additionally, any 16S PCR result must be interpreted within the clinical context, as any bacterial cellular material will be detected, including sample contaminants.

\section{Areas for further research}

Rapid identification and sensitivity testing for cultured organisms is an area of ongoing research. In the future, this will help to guide clinical decision-making in real time.

\section{CLINICAL BOTTOM LINE}

(See online supplemental infographic)

- Judicious use of BC should always be employed. Consider both diagnostic stewardship and pretest probability of BSI, alongside clinical assessment.

- Numerous factors impact on BC sensitivity, but adequate blood volume is of utmost importance.

- When interpreting a positive BC, adopt a holistic approach. Always ask, does this fit with the clinical picture?

- In persistent positive $\mathrm{BC}$, or where there is clinical concern, seek early advice from microbiology and infectious diseases specialists. Review the antimicrobial treatment and consider source identification and control.

- When pretest probability of BSI is high but no organism is cultured, consider 16S PCR testing. This can detect fastidious organisms, which can clinch an undiagnosed clinical syndrome.

\section{Twitter Peter Mallett @SimEdRBHSC}

Acknowledgements The team acknowledges colleagues in clinical microbiology and paediatrics within Royal Group of Hospitals. The authors thank Joseph Kelly and the medical illustrations team in Royal Victoria Hospital. Additionally, we thank Nathan Warnock for designing the article infographic.

Contributors PMa conceived the idea of update on the earlier version. $\mathrm{SOH}$ and PMa drafted and revised serial manuscript versions. PN provided microbiology expertise and edited the manuscript. LS and PMo reviewed and edited the manuscript. All contributed to the final review.

Funding The authors have not declared a specific grant for this research from any funding agency in the public, commercial or not-for-profit sectors.

\section{Competing interests None declared.}

Patient consent for publication Not required.

Provenance and peer review Commissioned; externally peer reviewed.

Supplemental material This content has been supplied by the author(s). It has not been vetted by BMJ Publishing Group Limited (BMJ) and may not have been peer-reviewed. Any opinions or recommendations discussed are solely those of the author(s) and are not endorsed by BMJ. BMJ disclaims all liability and responsibility arising from any reliance placed on the content. Where the content includes any translated material, BMJ does not warrant the accuracy and reliability of the translations (including but not limited to local regulations, clinical guidelines, terminology, drug names and drug dosages), and is not responsible for any error and/or omissions arising from translation and adaptation or otherwise.

\section{ORCID iD}

Peter Mallett http://orcid.org/0000-0003-2634-7785

\section{REFERENCES}

1 Dien Bard J, McElvania TeKippe E. Diagnosis of bloodstream infections in children. J Clin Microbiol 2016;54:1418-24.

2 Sharland M, Butler K, Giaquinto C. Chapter 26 Laboratory diagnosis of infection. In: Manual of childhood infections: the blue book. 4th ed. Oxford University Press, 2016.

3 ESPID Education Course Faculty. European Society for paediatric infectious diseases (ESPID) education, antibiotics management e-modules, 2019. Available: https://education. espid.org [Accessed 19 Nov 2020].

4 Munro A. Paediatric blood cultures- We're doing it wrong. Don't Forget the Bubbles 2019.

5 DFTB T, Munro A. Unlucky dip: rational diagnostic testing for infections. Don't Forget The Bubbles 2019.

6 Biondi EA, Mischler M, Jerardi KE, et al. Blood culture time to positivity in febrile infants with bacteremia. JAMA Pediatr 2014;168:844-9.

7 Garcia RA, Spitzer ED, Beaudry J, et al. Multidisciplinary team review of best practices for collection and handling of blood cultures to determine effective interventions for increasing the yield of true-positive bacteremias, reducing contamination, and eliminating false-positive central lineassociated bloodstream infections. Am J Infect Control 2015;43:1222-37.

8 Patel S, Cunney R, Demirjian A. Working Party for British Society for antimicrobial chemotherapyPaediatric Pathways. Available: https://bsac.org.uk/paediatricpathways/ [Accessed 21 Jan 2021].

9 Le Doare K, Nichols A-L, Payne H, et al. Very low rates of culture-confirmed invasive bacterial infections in a prospective 3-year population-based surveillance in Southwest London. Arch Dis Child 2014;99:526-31.

10 Neuman MI, Hall M, Lipsett SC, et al. Utility of blood culture among children hospitalized with community-acquired pneumonia. Pediatrics 2017;140:e20171013.

11 Michelson KA, Neuman MI, Pruitt CM, et al. Height of fever and invasive bacterial infection. Arch Dis Child 2021;106:5946.

12 Thé T, Curfman A, Burnham C-AD, et al. Pediatric anaerobic blood culture practices in Industrialized countries. J Appl Lab Med 2019;3:553-8.

13 Connell TG, Rele M, Cowley D, et al. How reliable is a negative blood culture result? volume of blood submitted for culture in routine practice in a children's Hospital. Pediatrics 2007;119:891-6.

14 Buttery JP. Blood cultures in newborns and children: optimising an everyday test. Arch Dis Child Fetal Neonatal Ed 2002;87:25F-8.

15 Isaacman DJ, Karasic RB, Reynolds EA, et al. Effect of number of blood cultures and volume of blood on detection of bacteremia in children. J Pediatr 1996;128:190-5.

16 Huber S, Hetzer B, Crazzolara R, et al. The correct blood volume for paediatric blood cultures: a conundrum? Clin Microbiol Infect 2020;26:168-73.

17 Mermel LA, Maki DG. Detection of bacteremia in adults: consequences of culturing an inadequate volume of blood. Ann Intern Med 1993;119:270-2.

18 Driscoll AJ, Deloria Knoll M, Hammitt LL, et al. The effect of antibiotic exposure and specimen volume on the detection of bacterial pathogens in children with pneumonia. Clin Infect Dis 2017;64:S368-77.

19 De SK, Shetty N, Kelsey M. How to use... blood cultures. Arch Dis Child Educ Pract Ed 2014;99:144-51. 
20 Baron EJ, Miller JM, Weinstein MP, et al. A guide to utilization of the microbiology laboratory for diagnosis of infectious diseases: 2013 recommendations by the Infectious Diseases Society of America (IDSA) and the American Society for Microbiology (ASM)(a). Clin Infect Dis 2013;57:e22-121.

21 Resti M, Micheli A, Moriondo M, et al. Comparison of the effect of antibiotic treatment on the possibility of diagnosing invasive pneumococcal disease by culture or molecular methods: a prospective, observational study of children and adolescents with proven pneumococcal infection. Clin Ther 2009;31:1266-73.

22 Kee PPL, Chinnappan M, Nair A, et al. Diagnostic yield of timing blood culture collection relative to fever. Pediatr Infect Dis J 2016;35:846-50.

23 UK Standards for Microbiology Investigations. Investigation of blood cultures (for organisms other than Mycobacterium species). Bacteriology;37:2.

24 Jardine LA, Sturgess BR, Inglis GDT, et al. Neonatal blood cultures: effect of delayed entry into the blood culture machine and bacterial concentration on the time to positive growth in a simulated model. J Paediatr Child Health 2009;45:210-4.

25 Dierig A, Berger C, Agyeman PKA, et al. Time-To-Positivity of blood cultures in children with sepsis. Front Pediatr 2018;6:222.

26 Hall KK, Lyman JA. Updated review of blood culture contamination. Clin Microbiol Rev 2006;19:788-802.

27 Hossain B, Islam MS, Rahman A, et al. Understanding bacterial isolates in blood culture and approaches used to define bacteria as contaminants: a literature review. Pediatr Infect Dis J 2016;35:S45-51.

28 Perez-Jorge EV, Burdette SD. Antibiotic therapy for positive blood culturesInfectious Disease and Antimicrobial Agents.
Available: http://www.antimicrobe.org/e38.asp\#t1 [Accessed 19 Nov 2020].

29 Murofushi Y, Furuichi M, Shoji K, et al. Adverse economic impact associated with blood culture contamination in a pediatric emergency department. Pediatr Infect Dis J 2018;37:755-8.

30 Habib G, Lancellotti P, Antunes MJ, et al. 2015 ESC guidelines for the management of infective endocarditis: the task force for the management of infective endocarditis of the European Society of cardiology (ESC). endorsed by: European association for Cardio-Thoracic surgery (EACTS), the European association of nuclear medicine (EANM). Eur Heart J 2015;36:3075-128.

31 Bhavsar SM, Dingle TC, Hamula CL. The impact of blood culture identification by MALDI-TOF MS on the antimicrobial management of pediatric patients. Diagn Microbiol Infect Dis 2018;92:220-5.

32 Rychert J. Benefits and limitations of MALDI-TOF mass spectrometry for the identification of microorganisms. $J$ Infectiology 2019;2:1-5.

33 Ray STJ, Drew RJ, Hardiman F, et al. Rapid identification of microorganisms by FilmArray blood culture identification panel improves clinical management in children. Pediatr Infect Dis J 2016;35:e134-8.

34 Rampini SK, Bloemberg GV, Keller PM, et al. Broad-Range $16 \mathrm{~S}$ rRNA gene polymerase chain reaction for diagnosis of culture-negative bacterial infections. Clin Infect Dis 2011;53:1245-51.

35 Stover KR, Morrison A, Collier T, et al. Epidemiology and risk factors for bacteremia in pediatric and adolescent patients. $J$ Pharm Pract 2019:089719001986805. 